\title{
Article
}

\section{A New Three-dimensional Assessment Model and Optimization for Acoustic Positioning System}

\author{
Lin Zhao ${ }^{1}$,Xiaobo Chen ${ }^{1, *}$, Yong Hao ${ }^{1}$, Chengcai Lv ${ }^{2}$, Lianhua Yu ${ }^{1}$ \\ 1 College of Automation, Harbin Engineering University, Harbin 150001, China; zhaolin@hrbeu.edu.cn(L.Z.); \\ haoyong@hrbeu.edu.cn(Y.H.); ruying0714@hotmail.com(L.Y.) \\ 2 Institute of Deep-sea Science and Engineering, Chinese Academy of Sciences, Sanya 572000, China; \\ lvchengcai@idsse.ac.cn(C.L.) \\ * Correspondence: cxiaobo@hotmail.com; Tel.: +86-157-6557-2980
}

\begin{abstract}
This paper addresses the problem of assessing and optimizing acoustic positioning system for underwater target localization with range measurements only. We present a new three-dimensional assessment model to assess the optimal geometric beacon formation whether meet user needs. For the sake of mathematical tractability, it is assumed that the measurements of the range between the target and beacons are corrupted with white Gaussian noise with variance is distance-dependent. Then by adopting dilution of precision (DOP) parameters in the assessment model, the relationship between DOP parameters and positioning accuracy is derived. In addition, the optimal geometric beacon formation that will yield the best performance is achieved by minimizing the values of geometric dilution of precision (GDOP) on condition that the position of target is known and fixed. Next, in order to make sure whether the estimate positioning accuracy over interesting region satisfy the precision needed by the users, geometric positioning accuracy (GPA), horizonal positioning accuracy (HPA) and vertical positioning accuracy (VPA) are utilized to assess the optimal geometric beacon formation. Simulation examples are designed to illustrate the exactness of the conclusion. Unlike other work which only use GDOP to optimize the formation and cannot assess the performance of the specified dimensions, this new three-dimensional assessment model can assess the optimal geometric beacon formation in each dimension for any point in three-dimensional space, which can provide users with guidance advices to optimize performance of every specified dimension.
\end{abstract}

Keywords: acoustic positioning system; three-dimensional assessment model; positioning accuracy; DOP; optimal configuration

\section{Introduction}

The last decade has witnessed tremendous progress in the development of marine technologies. Marine robotics, for example, autonomous underwater vehicle (AUV) are becoming ubiquitous in the execution of individual and military affairs. The reliable, accurate underwater positioning and navigation system is quite important to the operation of AUV. The underwater positioning and navigation system mainly includes inertial navigation system [1,2], geophysical navigation system $[3,4]$, visual navigation system $[5,6]$, acoustic positioning system $[7,8]$ and integrated navigation system $[9,10]$. To locate targets moving in a large range and for long time, acoustic positioning system is always utilized. There are lots of researchers have interest in solutions for the problem how to place the beacons in two or three-dimensional space. Hong Z. [11] addressed the problem of determining the optimal two-dimensional spatial placement of multiple sensors participating in a robot perception task. Levanon, N. [12] studied position determination in two-dimensional scenarios by achieving the lowest GDOP when range measured from beacons optimally located at the vertices of a regular n-sided polygon to the target. It is noteworthy that the definition of GDOP contains the fundamental relationship between measurement errors and computed position and time bias errors [13]. In this 
paper, the definition of dilution of precision (DOP) is similar to that in [13]. Moreno-Salinas et al. studied the multiple target localization with range measurements in unconstrained two-dimensional scenarios [14]. Some other work also paid attention to three-dimensional scenarios. In [15], the authors studied optimal sensor placement and motion coordination strategies for mobile sensor networks. They investigated the determinant of the fisher information matrix (FIM) in the two-dimensional and three-dimensional cases. The latter work was studied on the FIM and the maximization of its determinant, in order to determine the sensor configuration that yields the most accurate positioning [16]. Literature [17-20] also had close related work. More recently, in [21], Zou, Y.J. et al. assumed that range measurements have different weights depending on their value and took uncertainty of initial node position into consideration for the calculation of determinant of FIM. All the researches above focused on the optimal beacon configuration, few focused on the assessment models and rules of acoustic positioning system like assessment models and rules of global navigation satellite system (GNSS). For example, in [22], the assessment models and rules of GNSS interoperability with range measurements were previously presented by the author. Assessment parameters: DOP, navigation satellite system precision and navigation satellite system integrity were introduced to assess the GNSS performance. The work in [23] presented a study on GPS combined with Indian regional navigation satellite system (IRNSS) with DOP to measure the satellite-receiver geometry related to positioning accuracy. More recently, Swaszek, P. F. et al. analysed lower bounds DOP to allow users to assess how well their receivers are performing respecting the best possible performance, that will be useful for users to select satellites with multiple GNSS constellations, see [24] and the references therein.

Motivated by previous work in the area, we offer the analytic assessment model using DOP parameters related to the position accuracy for the problem of assessing acoustic positioning system in this paper. Then the geometric beacon placement is optimized based on target to beacons range measurements only. Next, what we should take care is the values of GPA, HPA and VPA over the interesting region where the sampling points taking place of the target. The document is organized as follows. In Section 2, DOP parameters and positioning accuracy for the assessment model are derived, the steps to assess the acoustic positioning system are listed as well. Section 3 contains the optimal beacon configurations for the case where the beacons can be placed freely in both two-dimensional scenario and three-dimensional scenario. The results of Section 3 are then examined in Section 4 for the particular scenario, and the steps to assess the acoustic positioning system in practice are shown. Finally, Section 5 contains the conclusions and further research.

\section{Assessment model: DOP parameters and position accuracy}

DOP parameters in literature [13] are defined as geometry factors that relate the target position errors to the measurements of the ranges errors. Generally, the receiver and beacons clock both have bias errors from the system time and the bias errors are a few microseconds or so, the speed of sound in water is about $1500 \mathrm{~m} / \mathrm{s}$, then the measurements errors are insignificant compared to the accuracy of positioning. Consequently, the DOP parameters in this paper don't consider the clock bias errors.

In what follows, the position of the $i$ th beacon is $\left(x_{i}, y_{i}, z_{i}\right)$ relative to the coordinate origin, and the target's actual position coordinates $(x, y, z)$ are considered unknown. In order to achieve the target's position in three dimensions, ideal measurements of ranges are made with $n(n>3)$ beacons from equations:

$$
d_{i}=f(x, y, z)=\sqrt{\left(x_{i}-x\right)^{2}+\left(y_{i}-y\right)^{2}+\left(z_{i}-z\right)^{2}}
$$

where $d_{i}$ is ideal measurement of range from the $i$ th beacon to the target without noise interference, $i$ ranges from 1 to $n$ and references the beacons.

However, the measurements are always corrupted by noise. We assume that all noise sources are independent and have equal variance, with this notation, the measurement model is given by:

$$
D_{i}=\sqrt{\left.\left(x_{i}-x\right)^{2}+\left(y_{i}-y\right)^{2}+\left(z_{i}-z\right)^{2}\right)}+\omega_{i}
$$


${ }_{81}$ where $D_{i}$ is actual measurement of range from the $i$ th beacon to the target with noise interference, $\omega_{i}$ is

82 the measurement error taken to be a zero mean Gaussian process $N\left(0, \sigma^{2}\right)$ with covariance is $\sigma^{2}$.

${ }_{83}$ Assuming that the target's position is approximately estimated as $\left(x_{e}, y_{e}, z_{e}\right)$ when the

84 measurements are corrupted by noise. However, we still use Equation (1) to estimate the position of

${ }_{85}$ target, thus we get the expression as follows:

$$
D_{i}=f\left(x_{e}, y_{e}, z_{e}\right)=\sqrt{\left(x_{i}-x_{e}\right)^{2}+\left(y_{i}-y_{e}\right)^{2}+\left(z_{i}-z_{e}\right)^{2}}
$$

86

${ }_{87}$ displacement $(\Delta x, \Delta y, \Delta z)$ described as:

$$
\begin{aligned}
& x=x_{e}+\Delta x \\
& y=y_{e}+\Delta y \\
& z=z_{e}+\Delta z
\end{aligned}
$$

88 Substituting (4) into (1), we can get the expression:

$$
f(x, y, z)=f\left(x_{e}+\Delta x, y_{e}+\Delta y, z_{e}+\Delta z\right)
$$

89 This latter function can be expanded about the approximate target's position using a Taylor series:

$$
\begin{aligned}
& f\left(x_{e}+\Delta x, y_{e}+\Delta y, z_{e}+\Delta z\right)=f\left(x_{e}, y_{e}, z_{e}\right)+ \\
& \frac{\partial f\left(x_{e}, y_{e}, z_{e}\right)}{\partial x_{e}} \Delta x+\frac{\partial f\left(x_{e}, y_{e}, z_{e}\right)}{\partial y_{e}} \Delta y+\frac{\partial f\left(x_{e}, y_{e}, z_{e}\right)}{\partial z_{e}} \Delta z+\cdots
\end{aligned}
$$

90

91 derivatives evaluate as follows:

$$
\begin{aligned}
& \frac{\partial f\left(x_{e}, y_{e}, z_{e}\right)}{\partial x_{e}}=-\frac{\left(x_{i}-x_{e}\right)}{D_{i}} \\
& \frac{\partial f\left(x_{e}, y_{e}, z_{e}\right)}{\partial y_{e}}=-\frac{\left(y_{i}-y_{e}\right)}{D_{i}} \\
& \frac{\partial f\left(x_{e}, y_{e}, z_{e}\right)}{\partial z_{e}}=-\frac{\left(z_{i}-z_{e}\right)}{D_{i}}
\end{aligned}
$$

By substituting (9) into (8), $\omega_{i}$ can be determined:

$$
\omega_{i}=D_{i}-d_{i}=a_{x i} \Delta x+a_{y i} \Delta y+a_{z i} \Delta z
$$

Now we have three unknowns composing the vector $\Delta \mathbf{u}=(\Delta x, \Delta y, \Delta z)^{\mathrm{T}}$ and the unknown 96 quantities can be determined by solving the matrix shown as: 


$$
\Delta \mathbf{d}=\mathbf{H} \Delta \mathbf{u}
$$

where $\mathrm{T}$ donates the transpose of matrix; the matrix $\Delta \mathbf{d}$ and observation matrix $\mathbf{H}$ are described by making the definitions:

$$
\begin{gathered}
\Delta \mathbf{d}=\left[\begin{array}{c}
\omega_{1} \\
\omega_{2} \\
\vdots \\
\omega_{n}
\end{array}\right] \\
\mathbf{H}=\left[\begin{array}{ccc}
a_{x 1} & a_{y 1} & a_{z 1} \\
a_{x 2} & a_{y 2} & a_{z 2} \\
\vdots & \vdots & \vdots \\
a_{x n} & a_{y n} & a_{z n}
\end{array}\right]
\end{gathered}
$$

If vectors $\left(a_{x i}, a_{y i}, a_{z i}\right)$ do not all lie in a plane, the weighting matrix $\left(\mathbf{H}^{\mathrm{T}} \mathbf{H}\right)$ will be invertible. Thus the method of least squares can be used to solve Equation (11) for $\Delta \mathbf{u}$ :

$$
\Delta \mathbf{u}=\left(\mathbf{H}^{\mathrm{T}} \mathbf{H}\right)^{-1} \mathbf{H}^{\mathrm{T}} \Delta \mathbf{d}
$$

In fact, the ideal positioning accuracy is decided by $\Delta \mathbf{u}$, then the covariance of $\Delta \mathbf{u}$ is obtained by forming the product $\Delta \mathbf{u} \Delta \mathbf{u}^{\mathrm{T}}$ and computing an expected value:

$$
\begin{aligned}
\operatorname{cov} \Delta \mathbf{u}=\mathrm{E}\left[\Delta \mathbf{u} \Delta \mathbf{u}^{\mathrm{T}}\right] & =\mathrm{E}\left[\left(\mathbf{H}^{\mathrm{T}} \mathbf{H}\right)^{-1} \mathbf{H}^{\mathrm{T}} \Delta \mathbf{d}\left(\left(\mathbf{H}^{\mathrm{T}} \mathbf{H}\right)^{-1} \mathbf{H}^{\mathrm{T}} \Delta \mathbf{d}\right)^{\mathrm{T}}\right] \\
& =\mathrm{E}\left[\left(\mathbf{H}^{\mathrm{T}} \mathbf{H}\right)^{-1} \mathbf{H}^{\mathrm{T}} \Delta \mathbf{d} \Delta \mathbf{d}^{\mathrm{T}} \mathbf{H}\left(\mathbf{H}^{\mathrm{T}} \mathbf{H}\right)^{-1}\right] \\
& =\left(\mathbf{H}^{\mathrm{T}} \mathbf{H}\right)^{-1} \mathbf{H}^{\mathrm{T}} \operatorname{cov}(\Delta \mathbf{d}) \mathbf{H}\left(\mathbf{H}^{\mathrm{T}} \mathbf{H}\right)^{-1}
\end{aligned}
$$

The usual assumption is that $\omega_{i}$ is distributed and independent, with zero mean Gaussian process whose variance is $\sigma^{2}$. The covariance of $\Delta \mathbf{d}$ is a scalar multiple of the identity: $\sigma^{2} \mathbf{I}_{n \times n}$, where $\mathbf{I}_{n \times n}$ is the $n \times n$ identity matrix.Then the result of Equation (15) is derived as:

$$
\operatorname{cov} \Delta \mathbf{u}=\sigma^{2}\left(\mathbf{H}^{\mathrm{T}} \mathbf{H}\right)^{-1} \mathbf{H}^{\mathrm{T}} \mathbf{H}\left(\mathbf{H}^{\mathrm{T}} \mathbf{H}\right)^{-1}=\sigma^{2}\left(\mathbf{H}^{\mathrm{T}} \mathbf{H}\right)^{-1}
$$

Under the stated assumptions, the covariance of the errors of the position is just a scalar multiple of the weighting matrix $\left(\mathbf{H}^{\mathrm{T}} \mathbf{H}\right)^{-1}$. The covariance of $\Delta \mathbf{u}$ is a $3 \times 3$ matrix and has an expanded representation:

$$
\operatorname{cov}(\Delta \mathbf{u})=\left[\begin{array}{ccc}
\sigma_{x}^{2} & \sigma_{x} \sigma_{y} & \sigma_{x} \sigma_{z} \\
\sigma_{x} \sigma_{y} & \sigma_{y}^{2} & \sigma_{y} \sigma_{z} \\
\sigma_{x} \sigma_{z} & \sigma_{y} \sigma_{z} & \sigma_{z}^{2}
\end{array}\right]
$$

The components of the weighting matrix $\left(\mathbf{H}^{\mathrm{T}} \mathbf{H}\right)^{-1}$ quantify how measurement errors translate into components of the covariance of $\Delta \mathbf{u}$. Express the weighting matrix $\left(\mathbf{H}^{\mathrm{T}} \mathbf{H}\right)^{-1}$ in component form:

$$
\left(\mathbf{H}^{\mathrm{T}} \mathbf{H}\right)^{-1}=\mathbf{D}=\left[\begin{array}{lll}
D_{11} & D_{12} & D_{13} \\
D_{21} & D_{22} & D_{23} \\
D_{31} & D_{32} & D_{33}
\end{array}\right]
$$

unlike other work which only use GDOP to optimize the formation and cannot assess the performance of any specified dimensions, more DOP parameters are presented in this paper, we 
use GDOP, HDOP and VDOP to assess the optimal geometric beacon formation in each dimension for any point in three-dimensional space:

$$
\begin{aligned}
& \mathrm{GDOP}=\sqrt{D_{11}+D_{22}+D_{33}}=\frac{\sqrt{\sigma_{x}^{2}+\sigma_{y}^{2}+\sigma_{z}^{2}}}{\sigma} \\
& \mathrm{HDOP}=\sqrt{D_{11}+D_{22}}=\frac{\sqrt{\sigma_{x}^{2}+\sigma_{y}^{2}}}{\sigma} \\
& \mathrm{VDOP}=\sqrt{D_{33}}=\frac{\sqrt{\sigma_{z}^{2}}}{\sigma}
\end{aligned}
$$

To assess the acoustic positioning system, the sampling points over interesting region are adopted to take place of the approximate target's position. Then the observation matrix $\mathbf{H}$ is achieved by the Equation (20):

$$
\mathbf{H}=\left[\begin{array}{ccc}
\frac{\left(x_{1}-x_{s}\right)}{r_{1}} & \frac{\left(y_{1}-y_{s}\right)}{r_{1}} & \frac{\left(z_{1}-z_{s}\right)}{r_{1}} \\
\frac{\left(x_{2}-x_{s}\right)}{r_{2}} & \frac{\left(y_{2}-y_{s}\right)}{r_{2}} & \frac{\left(z_{2}-z_{s}\right)}{r_{2}} \\
\vdots & \vdots & \vdots \\
\frac{\left(x_{n}-x_{s}\right)}{r_{n}} & \frac{\left(y_{n}-y_{s}\right)}{r_{n}} & \frac{\left(z_{n}-z_{s}\right)}{r_{n}}
\end{array}\right]
$$

where $\left(x_{i}, y_{i}, z_{i}\right), i=1 \cdots n$ denote the $i$ th beacon's position in three dimensions, $\left(x_{s}, y_{s}, z_{s}\right)$ denote the sampling point's position over interesting region in three dimensions, $r_{i}$ is the range between the $i$ th beacon and the sampling point.

Equation (20) is valid provided that the range measurement errors are sufficiently small so that the error between sample point's actual position and approximately estimated position can be ignored. The minimum of $\sigma$ is $c / 2 f$ in theory, where $c$ is the speed of propagation of sound in the water, $f$ is the frequency of sound in the water. However, $\sigma$ in practice is always far larger than $c / 2 f$. Multiply $\sigma$ by GDOP, HDOP and VDOP, respectively, then the GPA, HPA and VPA will be obtained, correspondingly.

$$
\begin{aligned}
& \mathrm{GPA}=\sqrt{\sigma_{x}^{2}+\sigma_{y}^{2}+\sigma_{z}^{2}}=\mathrm{GDOP} \times \sigma \\
& \mathrm{HPA}=\sqrt{\sigma_{x}^{2}+\sigma_{y}^{2}}=\mathrm{HDOP} \times \sigma \\
& \mathrm{VPA}=\sigma_{z}=\mathrm{VDOP} \times \sigma
\end{aligned}
$$

Compare the GPA, HPA and VPA with the user requirements and decide whether the positioning accuracy meet the user requirements. If they don't, that means more beacons are needed. If the HPA don't satisfy the user requirements, add the beacons in the horizonal plane. If the VPA don't satisfy the user requirements, add the beacons in vertical plane. If the GPA don't satisfy the user requirements, add the beacons in horizonal plane mainly.

According to the above analysis, the steps to assessment acoustic positioning system can be summarized as follows:

1. Determine the optimal beacons configurations with $n$ beacons: the beacons should be distributed at the vertices of a regular n-sided polygon on the same plane. This conclusion is drawn in Section 3.

2. According to the spatial resolution needed by users, make sure the sampling points in space, then compute the ranges from beacons to the all sampling points in space by Equation (1).

3. Define the variance of range measurements errors $\sigma$, obtain the DOP parameters, GDOP, HDOP and VDOP by the Equation (19), and compute the GPA, HPA and VPA by the Equation (21).

4. Define the variance of range measurements errors $\sigma$, obtain the DOP parameters, GDOP, HDOP and VDOP by the Equation (19), and compute the GPA, HPA and VPA by the Equation (21).

5. Determine whether increase the number of beacons into the acoustic positioning system according to the compare between the ideal positioning accuracy and the users' requirements. 


\section{The optimal beacons configurations with DOP parameters}

\subsection{Two-dimensional scenarios}

This section addresses the problem of estimate beacon placement for underwater target positioning in two-dimensional space, subject to the condition that the beacons and the sampling point lie on the horizontal plane. In this situation, $\mathbf{H}$ is singular so the matrix $\mathbf{D}$ doesn't exist. For the sake of simplicity, and without loss of generality, the sampling point is considered to be located at the origin of the inertial coordinate frame hereinafter. It is assumed that the position of the $i$ th beacon is located on the point whose radius is $r_{i}$ and the polar angle is $\alpha_{i}$, so the polar coordinates of the $i$ th beacon is $\left(r_{i} \cos \alpha_{i}, r_{i} \sin \alpha_{i}\right)$, then the matrix $\mathbf{H}$ can be simplified as $\mathbf{H}_{\mathbf{1}}$ :

$$
\mathbf{H}_{\mathbf{1}}=\left[\begin{array}{cc}
\frac{r_{1} \cos \alpha_{1}}{r_{1}} & \frac{r_{1} \sin \alpha_{1}}{r_{1}} \\
\frac{r_{2} \cos \alpha_{2}}{r_{2}} & \frac{r_{2} \sin \alpha_{2}}{r_{2}} \\
\vdots & \vdots \\
\frac{r_{n} \cos \alpha_{n}}{r_{n}} & \frac{r_{n} \sin \alpha_{n}}{r_{n}}
\end{array}\right]=\left[\begin{array}{cc}
\cos \alpha_{1} & \sin \alpha_{1} \\
\cos \alpha_{2} & \sin \alpha_{2} \\
\vdots & \vdots \\
\cos \alpha_{n} & \sin \alpha_{n}
\end{array}\right]
$$

At this point, we introduce the vectors, $\mathbf{X}$ and $\mathbf{Y}$, defined as:

$$
\begin{aligned}
& \mathbf{X}=\left[\begin{array}{llll}
\cos \alpha_{1} & \cos \alpha_{2} & \cdots & \cos \alpha_{n}
\end{array}\right] \\
& \mathbf{Y}=\left[\begin{array}{llll}
\sin \alpha_{1} & \sin \alpha_{2} & \cdots & \sin \alpha_{n}
\end{array}\right]
\end{aligned}
$$

It's obvious that the analytical relationship of the determinant of $\mathbf{X}$ and $\mathbf{Y}$ is as follows:

$$
|\mathbf{X}|^{2}+|\mathbf{Y}|^{2}=n
$$

As a consequence, define $\phi$ is the angle formed by vectors $\mathbf{X}$ and $\mathbf{Y}$, then the weighting matrix $\mathbf{D}_{\mathbf{1}}=\left(\mathbf{H}_{\mathbf{1}}{ }^{\mathrm{T}} \mathbf{H}_{\mathbf{1}}\right)^{-1}$ is parameterized by two vectors $\mathbf{X}$ and $\mathbf{Y}$ :

$$
\mathbf{D}_{\mathbf{1}}=\left[\begin{array}{cc}
\sum_{i=1}^{n} \cos ^{2} \alpha_{i} & \sum_{i=1}^{n} \cos \alpha_{i} \sin \alpha_{i} \\
\sum_{i=1}^{n} \cos \alpha_{i} \sin \alpha_{i} & \sum_{i=1}^{n} \sin ^{2} \alpha_{i}
\end{array}\right]^{-1}=\frac{\left[\begin{array}{cc}
\mathbf{Y}^{2} & -|\mathbf{X}||\mathbf{Y}| \cos \phi \\
-|\mathbf{X}||\mathbf{Y}| \cos \phi & \mathbf{X}^{2}
\end{array}\right]}{\operatorname{det}\left(\mathbf{H}_{\mathbf{1}}{ }^{\mathrm{T}} \mathbf{H}_{\mathbf{1}}\right)}
$$

The determinant of $\mathbf{H}_{\mathbf{1}}{ }^{\mathrm{T}} \mathbf{H}_{\mathbf{1}}$ yields:

$$
\operatorname{det}\left(\mathbf{H}_{\mathbf{1}}{ }^{\mathrm{T}} \mathbf{H}_{\mathbf{1}}\right)=|\mathbf{X}|^{2}|\mathbf{Y}|^{2}\left(1-\cos ^{2} \phi\right)
$$

Obviously, $\cos ^{2} \phi=0$ is the only feasible solution to make the $\operatorname{det}\left(\mathbf{H}_{\mathbf{1}}{ }^{\mathrm{T}} \mathbf{H}_{\mathbf{1}}\right)$ largest that implies that vectors $\mathbf{X}$ and $\mathbf{Y}$ are orthogonal. This condition makes $\left(\mathbf{H}_{\mathbf{1}}{ }^{\mathrm{T}} \mathbf{H}_{\mathbf{1}}\right)^{-1}$ be a diagonal matrix and $\operatorname{det}\left(\mathbf{H}_{\mathbf{1}}{ }^{\mathrm{T}} \mathbf{H}_{\mathbf{1}}\right)$ can now be written as:

$$
\operatorname{det}\left(\mathbf{H}_{\mathbf{1}}{ }^{\mathrm{T}} \mathbf{H}_{\mathbf{1}}\right)=|\mathbf{X}|^{2}|\mathbf{Y}|^{2}=|\mathbf{X}|^{2}\left(n-|\mathbf{X}|^{2}\right) \leq-\left(|\mathbf{X}|^{2}-n / 2\right)^{2}+n^{2} / 4
$$

One obtains, finally:

$$
\mathrm{HDOP} \geq \sqrt{\frac{n}{n^{2} / 4}}=\sqrt{\frac{4}{n}}
$$

We will now make sure the beacon configurations. Define the sampling point at the center of an n-sided regular polygon $(n \geq 2)$, and the $n$ beacons are placed at the vertices of a regular $n$-sided polygon. Then coordinate of the $i$ th beacons is described as: 


$$
\left(r_{i} \cos \frac{2 \pi(i-1)}{n}, r_{i} \sin \frac{2 \pi(i-1)}{n}\right) i=1,2, \cdots, n
$$

Then the matrix $\mathbf{H}_{\mathbf{1}}$ can be described as:

$$
\mathbf{H}_{\mathbf{1}}=\left[\begin{array}{cc}
\cos 0 & \sin 0 \\
\cos \frac{2 \pi}{n} & \sin \frac{2 \pi}{n} \\
\vdots & \vdots \\
\cos \frac{2 \pi(i-1)}{n} & \sin \frac{2 \pi(i-1)}{n}
\end{array}\right]
$$

Using the Fourier summation formulas:

$$
\begin{aligned}
& \sum_{i=1}^{n} \cos ^{2} \frac{2 \pi(i-1)}{n}=\frac{n}{2} \\
& \sum_{i=1}^{n} \sin ^{2} \frac{2 \pi(i-1)}{n}=\frac{n}{2} \\
& \sum_{i=1}^{n} \cos \frac{2 \pi(i-1)}{n} \sin \frac{2 \pi(i-1)}{n}=0 \\
& \sum_{i=1}^{n} \cos \frac{2 \pi(i-1)}{n}=\sum_{i=1}^{n} \sin \frac{2 \pi(i-1)}{n}=0
\end{aligned}
$$

Substituting Equation (31) into Equation (25) yields:

$$
\mathbf{D}_{1}=\left[\begin{array}{cc}
\frac{n}{2} & 0 \\
0 & \frac{n}{2}
\end{array}\right]^{-1}=\left[\begin{array}{cc}
\frac{2}{n} & 0 \\
0 & \frac{2}{n}
\end{array}\right]
$$

We now can draw the conclusion that in two-dimensional scenarios, it is clear that the beacon configurations have no explicit dependence on the ranges, only related to the angles that the range vectors form with the unit axes of the frame. What's more, for position determination, based on $\mathrm{n}$ range measurements $(n>2)$, the lowest possible HDOP is $2 / \sqrt{n}$. This value will occur when the sampling point is on the initial point, and the $n$ beacons are located at the vertices of regular $n$-sided polygon. Then more optimal beacon configurations can be generated by two methods: 1) multiplying the range of each beacon to the sampling point by an arbitrary positive number as long as the sampling point could receive the signals from all beacons. 2) rotating the beacon formation rigidly in terms of an arbitrary axis. However, the two methods can only make the lowest possible HDOP constant when the sampling point is on the initial point, and the $n$ beacons are located at the vertices of regular $n$-sided polygon.

\subsection{Three-dimensional scenarios}

Similar to the two-dimensional scenarios, the sampling point is considered to be located at the origin of the inertial coordinate frame hereinafter. Assume that the position of the $i$ th beacon is located on the point $\left(x_{i}, y_{i}, z_{i}\right)$, and the range between the sampling point and the beacon is $r_{i}=$ $\sqrt{\left(x_{i}^{2}+y_{i}^{2}+z_{i}^{2}\right)}$. Define the angles $\left(\alpha_{i}, \beta_{i}, \gamma_{i}\right)$ shown as Equation (33):

$$
\begin{aligned}
\cos \alpha_{i} & =x_{i} / r_{i} \\
\cos \beta_{i} & =y_{i} / r_{i} \\
\cos \gamma_{i} & =z_{i} / r_{i}
\end{aligned}
$$


Then the coordinate of the $i$ th beacon can be defined as $\left(r_{i} \cos \alpha_{i}, r_{i} \cos \beta_{i}, r_{i} \cos \gamma_{i}\right)$. The matrix of $\mathbf{H}$ becomes:

$$
\mathbf{H}_{\mathbf{1}}=\left[\begin{array}{ccc}
\frac{r_{1} \cos \alpha_{1}}{r_{1}} & \frac{r_{1} \cos \beta_{1}}{r_{1}} & \frac{r_{1} \cos \gamma_{1}}{r_{1}} \\
\frac{r_{2} \cos \alpha_{2}}{r_{2}} & \frac{r_{2} \cos \beta_{2}}{r_{2}} & \frac{r_{2} \cos \gamma_{2}}{r_{2}} \\
\vdots & \vdots & \vdots \\
\frac{r_{n} \cos \alpha_{n}}{r_{n}} & \frac{r_{n} \cos \beta_{n}}{r_{n}} & \frac{r_{n} \cos \gamma_{n}}{r_{n}}
\end{array}\right]=\left[\begin{array}{ccc}
\cos \alpha_{1} & \cos \beta_{1} & \cos \gamma_{1} \\
\cos \alpha_{2} & \cos \beta_{2} & \cos \gamma_{2} \\
\vdots & \vdots & \vdots \\
\cos \alpha_{n} & \cos \beta_{n} & \cos \gamma_{n}
\end{array}\right]
$$

It is convenient to introduce the vectors $\mathbf{X}, \mathbf{Y}$ and $\mathbf{Z}$ defined as:

$$
\begin{aligned}
\mathbf{X} & =\left[\begin{array}{llll}
\cos \alpha_{1} & \cos \alpha_{2} & \cdots & \cos \alpha_{n}
\end{array}\right] \\
\mathbf{Y} & =\left[\begin{array}{llll}
\cos \beta_{1} & \cos \beta_{2} & \cdots & \cos \beta_{n}
\end{array}\right] \\
\mathbf{Z} & =\left[\begin{array}{llll}
\cos \gamma_{1} & \cos \gamma_{2} & \cdots & \cos \gamma_{n}
\end{array}\right]
\end{aligned}
$$

The relationship of the determinant of $\mathbf{X}, \mathbf{Y}$ and $\mathbf{Z}$ is shown as follows:

$$
|\mathbf{X}|^{2}+|\mathbf{Y}|^{2}+|\mathbf{Z}|^{2}=n
$$

Computations show that Equation (18) can be rewritten as:

$$
\mathbf{D}=\left(\mathbf{H}^{\mathrm{T}} \mathbf{H}\right)^{-1}=\left[\begin{array}{ccc}
|\mathbf{X}|^{2} & |\mathbf{X}||\mathbf{Y}| \cos \varphi & |\mathbf{X}||\mathbf{Z}| \cos \theta \\
|\mathbf{X}||\mathbf{Y}| \cos \varphi & |\mathbf{Y}|^{2} & |\mathbf{Y}||\mathbf{Z}| \cos \omega \\
|\mathbf{X}||\mathbf{Z}| \cos \theta & |\mathbf{Y}||\mathbf{Z}| \cos \omega & |\mathbf{Z}|^{2}
\end{array}\right]^{-1}
$$

where $\varphi, \theta$ and $\omega$ are the angles formed by vectors $\mathbf{X}$ and $\mathbf{Y}, \mathbf{Y}$ and $\mathbf{Z}, \mathbf{Y}$ and $\mathbf{Z}$, respectively. From Equation (37) it follows that:

$$
\text { GDOP }=\sqrt{\frac{|\mathbf{Y}|^{2}|\mathbf{Z}|^{2}\left(1-\cos ^{2} \omega\right)+|\mathbf{X}|^{2}|\mathbf{Z}|^{2}\left(1-\cos ^{2} \theta\right)+|\mathbf{X}|^{2}|\mathbf{Y}|^{2}\left(1-\cos ^{2} \varphi\right)}{\operatorname{det}\left(\mathbf{H}^{\mathrm{T}} \mathbf{H}\right)}}
$$

The determinant of $\left(\mathbf{H}^{\mathrm{T}} \mathbf{H}\right)$ yields:

$$
\operatorname{det}\left(\mathbf{H}^{\mathrm{T}} \mathbf{H}\right)=|\mathbf{X}|^{2}|\mathbf{Y}|^{2}|\mathbf{Z}|^{2}\left(1+2 \cos \omega \cos \theta \cos \varphi-\cos ^{2} \theta-\cos ^{2} \omega-\cos ^{2} \varphi\right)
$$

We suppose a procedure inspired in the two-dimensional problem, the optimal solution is:

$$
\cos \omega=\cos \theta=\cos \varphi=0
$$

In this situation, it follows that:

$$
\begin{aligned}
& \frac{1-\cos ^{2} \omega}{1+2 \cos \omega \cos \theta \cos \varphi-\cos ^{2} \theta-\cos ^{2} \omega-\cos ^{2} \varphi}=1 \\
& \frac{1-\cos ^{2} \theta}{1+2 \cos \omega \cos \theta \cos \varphi-\cos ^{2} \theta-\cos ^{2} \omega-\cos ^{2} \varphi}=1 \\
& \frac{1-\cos ^{2} \varphi}{1+2 \cos \omega \cos \theta \cos \varphi-\cos ^{2} \theta-\cos ^{2} \omega-\cos ^{2} \varphi}=1
\end{aligned}
$$

We now show that 1 is their minimum possible values. Without loss of generality, suppose that a smaller value that clearly satisfies: 


$$
\frac{1-\cos ^{2} \omega}{1+2 \cos \omega \cos \theta \cos \varphi-\cos ^{2} \theta-\cos ^{2} \omega-\cos ^{2} \varphi}<1
$$

As it's known to all, the determinant of symmetrical matrix $\mathbf{H}^{\mathrm{T}} \mathbf{H}$ is not less than 0 . What's more, GDOP is inexistence when the determinant of $\mathbf{H}^{\mathrm{T}} \mathbf{H}=0$. Therefore, the determinant of $\mathbf{H}^{\mathrm{T}} \mathbf{H}>0$ is in consideration. In this situation, the above inequality is equivalent to:

$$
0<2 \cos \omega \cos \theta \cos \varphi-\cos ^{2} \theta-\cos ^{2} \varphi
$$

Because $|\cos \omega|<1$, it follows that:

$$
2 \cos \omega \cos \theta \cos \varphi \leq \cos ^{2} \theta+\cos ^{2} \varphi
$$

This conclusion contradicts Equation (43). Therefore:

$$
\frac{1-\cos ^{2} \omega}{1+2 \cos \omega \cos \theta \cos \varphi-\cos ^{2} \theta-\cos ^{2} \omega-\cos ^{2} \varphi} \geq 1
$$

Similarly, we can prove that:

$$
\begin{gathered}
\frac{1-\cos ^{2} \theta}{1+2 \cos \omega \cos \theta \cos \varphi-\cos ^{2} \theta-\cos ^{2} \omega-\cos ^{2} \varphi} \geq 1 \\
\frac{1-\cos ^{2} \varphi}{1+2 \cos \omega \cos \theta \cos \varphi-\cos ^{2} \theta-\cos ^{2} \omega-\cos ^{2} \varphi} \geq 1
\end{gathered}
$$

In these circumstances, GDOP is computed as:

$$
\mathrm{GDOP}=\sqrt{\frac{1}{|\mathbf{X}|^{2}}+\frac{1}{|\mathbf{Y}|^{2}}+\frac{1}{|\mathbf{Z}|^{2}}}=\sqrt{\frac{1}{|\mathbf{X}|^{2}}+\frac{1}{|\mathbf{Y}|^{2}}+\frac{1}{n-|\mathbf{X}|^{2}-|\mathbf{Y}|^{2}}}
$$

Construct the binary function $f(a, b)$ as follows:

$$
f(a, b)=\frac{1}{a}+\frac{1}{b}+\frac{1}{n-a-b}
$$

The Hessian matrix of Equation (48) is:

$$
\text { Hessian }=\left[\begin{array}{ll}
\frac{\partial^{2} f(a, b)}{\partial a^{2}} & \frac{\partial f(a, b)}{\partial a \partial b} \\
\frac{\partial f(a, b)}{\partial b \partial a} & \frac{\partial^{2} f(a, b)}{\partial b^{2}}
\end{array}\right]=\left[\begin{array}{cc}
\frac{2}{a^{3}}+\frac{2}{(n-a-b)^{3}} & \frac{2}{(n-a-b)^{3}} \\
\frac{2}{(n-a-b)^{3}} & \frac{2}{a^{3}}+\frac{2}{(n-a-b)^{3}}
\end{array}\right]
$$

where it is easy to proof that the Hessian matrix of Equation (48) is positive definite, the minimum value of $f(a, b)$ is obtained provided that the first derivatives equal to 0 :

$$
\begin{aligned}
& \frac{\partial f(a, b)}{\partial a}=\frac{1}{(n-a-b)^{2}}-\frac{1}{a^{2}}=0 \\
& \frac{\partial f(a, b)}{\partial a}=\frac{1}{(n-a-b)^{2}}-\frac{1}{b^{2}}=0
\end{aligned}
$$

From which follows that:

$$
a=b=\frac{n}{3}
$$

Substituting this result in Equation (36), we obtain: 


$$
|\mathbf{X}|^{2}=|\mathbf{Y}|^{2}=|\mathbf{Z}|^{2}=\frac{n}{3}
$$

Now we determine the geometric configuration in three-dimensional scenarios. To simplify the computation, we assume that the optimal beacon formations are placed on a unit sphere centered at the sampling point. Inspired by the work in two-dimensional scenarios, we address the problem of optimal beacon placement subject to the condition that the beacons lie on the same plane. Then the beacons may be distributed at the vertices of a regular n-sided polygon, which belongs to the circumference of the plane $z=\frac{1}{\sqrt{3}}$ or on the circumference of the plane $z=-\frac{1}{\sqrt{3}}$. In addition, the optimal radius $R=\sqrt{\frac{2}{3}}$. Now we give a simple proof of this geometric configuration. Firstly, rewrite the positions of the beacons in polar coordinates as:

$$
\begin{aligned}
\cos \alpha_{i} & =x_{i} / r_{i}=x_{i} \\
\cos \beta_{i} & =y_{i} / r_{i}=y_{i} \\
\cos \gamma_{i} & =z_{i} / r_{i}=z_{i}
\end{aligned}
$$

Because all beacons are distributed on the circumference of the plane $z=\frac{1}{\sqrt{3}}$ or on the circumference of the plane $z=-\frac{1}{\sqrt{3}}$. It then follows that:

$$
\begin{aligned}
& \cos ^{2} \gamma_{i}=\frac{1}{3} \\
& |\mathbf{Z}|^{2}=\sum_{i=1}^{n} \cos ^{2} \gamma_{i}=\frac{n}{3}
\end{aligned}
$$

With the conclusion in Equation (31), we can get:

$$
\begin{aligned}
& \sum_{i=1}^{n}\left(\frac{x_{i}}{R}\right)^{2}=\sum_{i=1}^{n} \cos ^{2} \frac{2 \pi(i-1)}{n}=\frac{n}{2} \\
& \sum_{i=1}^{n}\left(\frac{y_{i}}{R}\right)^{2}=\sum_{i=1}^{n} \sin ^{2} \frac{2 \pi(i-1)}{n}=\frac{n}{2} \\
& \sum_{i=1}^{n}\left(\frac{x_{i}}{R}\right)\left(\frac{y_{i}}{R}\right)=\sum_{i=1}^{n} \cos \frac{2 \pi(i-1)}{n} \sin \frac{2 \pi(i-1)}{n}=0 \\
& \sum_{i=1}^{n}\left(\frac{x_{i}}{R}\right)=\sum_{i=1}^{n} \cos \frac{2 \pi(i-1)}{n}=0 \\
& \sum_{i=1}^{n}\left(\frac{y_{i}}{R}\right)=\sum_{i=1}^{n} \sin \frac{2 \pi(i-1)}{n}=0
\end{aligned}
$$

Therefore, we can obtain the formula as follows: 


$$
\begin{aligned}
& |\mathbf{X}|^{2}=\sum_{i=1}^{n}\left(x_{i}\right)^{2}=\frac{n}{2} \times R^{2}=\frac{n}{3} \\
& |\mathbf{Y}|^{2}=\sum_{i=1}^{n}\left(y_{i}\right)^{2}=\frac{n}{2} \times R^{2}=\frac{n}{3} \\
& |\mathbf{X}||\mathbf{Y}|=\sum_{i=1}^{n}\left(x_{i} y_{i}\right)=0 \\
& |\mathbf{Z}||\mathbf{X}|=\sqrt{\frac{1}{3}} \sum_{i=1}^{n}\left(x_{i}\right)=0 \\
& |\mathbf{Z}||\mathbf{Y}|=\sqrt{\frac{1}{3}} \sum_{i=1}^{n}\left(y_{i}\right)=0
\end{aligned}
$$

Then Equation (37) yields:

$$
\mathbf{D}=\left(\mathbf{H}^{\mathrm{T}} \mathbf{H}\right)^{-1}=\left[\begin{array}{ccc}
\frac{n}{3} & 0 & 0 \\
0 & \frac{n}{3} & 0 \\
0 & 0 & \frac{n}{3}
\end{array}\right]^{-1}=\left[\begin{array}{ccc}
\frac{3}{n} & 0 & 0 \\
0 & \frac{3}{n} & 0 \\
0 & 0 & \frac{3}{n}
\end{array}\right]
$$

Once the optimal beacon placement on a unit sphere in three-dimensional scenarios is found in terms of the direction cosines achieved above, more infinite optimal beacon placements can be generated by multiplying the range of each beacon to the sampling point by an arbitrary positive number, as long as the sampling point can receive the signal from the beacons. The lowest possible GDOP is $3 / \sqrt{n}$ based on $n$ range measurements $(n>3)$.

An interesting problem arises: whether HDOP and VDOP get the lowest value when the optimal beacons configurations make GDOP lowest. The answer is no. Assume that the coordinate of the $i$ th beacon is $\left(x_{i}, y_{i}, z\right)$, without loss of generality, if the beacons are located on a circle centered at the sampling point described as:

$$
x_{i}^{2}+y_{i}^{2}=\hat{r}^{2}
$$

With the condition shown in Equations (36) and (40), HDOP and VDOP are described as:

$$
\begin{gathered}
\mathrm{HDOP}=\sqrt{\frac{1}{|\mathbf{X}|^{2}}+\frac{1}{|\mathbf{Y}|^{2}}}=\sqrt{\frac{n-|\mathbf{Z}|^{2}}{|\mathbf{X}|^{2}|\mathbf{Y}|^{2}}}=\sqrt{\frac{n-n \frac{z^{2}}{\sum_{i=1}^{n} \frac{x_{i}^{2}}{\hat{r}^{2}+z^{2}} \sum_{i=1}^{n} \frac{y_{i}^{2}}{\hat{r}^{2}+z^{2}}}}{\sum_{i=1}^{n} x_{i}^{2} \sum_{i=1}^{n} y_{i}^{2}}}=\sqrt{\frac{\left.n\left(\hat{r}^{2}+z^{2}\right)^{2}-n \hat{r}^{2}+z^{2}\right)}{\sum_{i=1}^{n} x_{i}^{2} \sum_{i=1}^{n} y_{i}^{2}}} \\
\operatorname{VDOP}=\sqrt{\frac{1}{|\mathbf{Z}|^{2}}}=\sqrt{\frac{1}{n \frac{z^{2}}{z^{2}+\hat{r}^{2}}}}=\sqrt{\frac{1}{n}\left(1+\frac{\hat{r}^{2}}{z^{2}}\right)}
\end{gathered}
$$

It's shown that the vertical distance $z$ between beacons and sampling point becomes larger, HDOP will be larger, and VDOP will be smaller. In many practical applications of interest, however, the sampling point's depth can be measured directly with small error. Thus there's no need to estimate it with acoustic range measurements. Therefore, based on the GDOP or HDOP, we can decide whether the beacons configuration meet user needs. 


\section{4}

\section{Simulation example}

\subsection{Optimal beacon placement in two-dimensional scenarios}

If there is only one sampling point in the acoustic positioning system and known to users, section 3.1 shows clearly that the optimal beacons are placed at the vertices of regular n-sided polygon. Given the experimental conditions, it's necessary to estimate how good the positioning accuracy in term of the beacon formation for anywhere in the acoustic positioning system. In order to achieve the goal, HPA with hypothetical sampling point on a grid in a finite spatial region $A$ is computed. In this paper, region $A$ will always be rectangle. The formation is the one in which four beacons are placed at $p_{1}=[2000,2000] m, p_{2}=[-2000,2000] m, p_{3}=[-2000,-2000] m, p_{4}=[2000,-2000] m$. It's assumed that all range measurements are corrupted by additive zero mean Gaussian noise with variance $\sigma=1$ so that the values of HPA are equal to that of HDOP. The spatial resolution chosen is $2 m \times 2 m$.

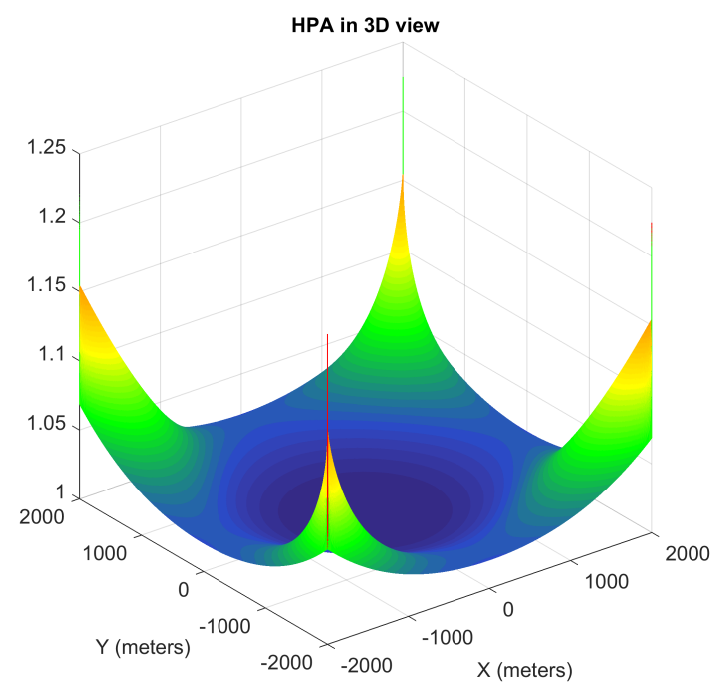

Figure 1. HPA in the 3D view in two-dimensional scenarios.

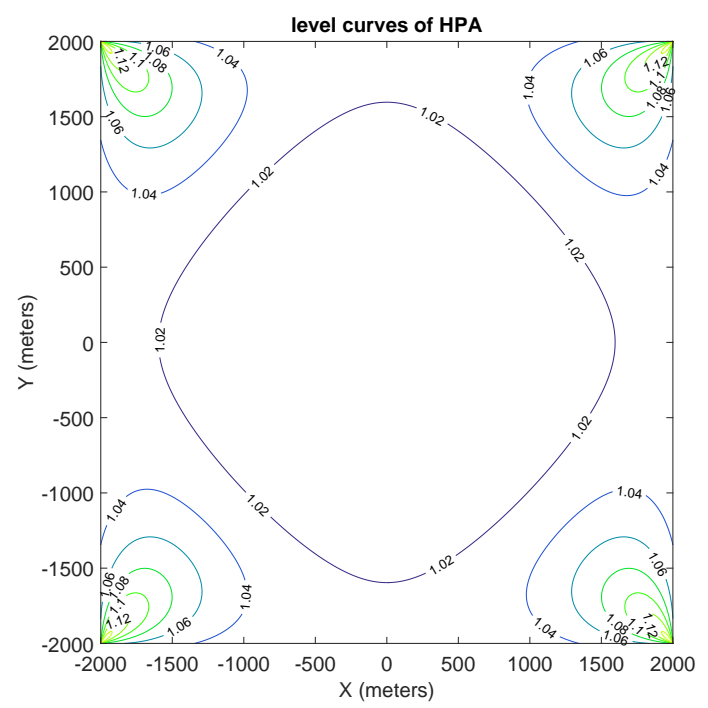

Figure 2. Level curves of HPA in two-dimensional scenarios. 
The simulation results of HPA are presented in Figure 1 and 2. In Figure 1, the values of HPA in 3D view for region $A$ are shown. It's important to note that the HPA obtained at the point of beacon are computed with three other beacons, without the beacon location itself, so as to avoid $\operatorname{det}\left(\mathbf{H}^{\mathrm{T}} \mathbf{H}\right)=0$. Thus, the values of HPA at these points are extra-large. In Figure 2, level curves of HPA in region $A$ are shown. We can draw the conclusion that better performance obtained in two-dimensional scenarios is within the circle of 1.02, which inspires us around the centre of the beacon is more accurate than that nearby the beacon. Furthermore, the better positioning region in two-dimensional scenarios is like a square, however, rotating 90 degrees to the beacon formation.

\subsection{Optimal beacon placement in three-dimensional scenarios}

For three-dimensional scenario, the beacons are supposed to be placed on the surface of the sea, and the coordinates are $p_{1}=[2000,2000,0] m, p_{2}=[-2000,2000,0] m, p_{3}=[-2000,-2000,0] m$, $p_{4}=[2000,-2000,0] \mathrm{m}$. Similar to the two-dimensional scenario, all range measurements are assumed to be corrupted by additive zero mean Gaussian noise with variance $\sigma=1$. Three different levels of sampling points' height are considered for the computation of the GPA involved in the optimization figuration. The depths of the sampling points are assumed to be $-1000 m,-2000 m$ and $-3000 m$, respectively. The spatial resolution chosen is $2 m \times 2 m$. In Figure 3 and 4, GPA values of the $-2000 m$ sampling points are supplied. The other GPA values of the $-1000 \mathrm{~m}$ and $-3000 \mathrm{~m}$ sampling points are similar to those of $-2000 \mathrm{~m}$. They aren't presented in this paper due to space limitations. The comparison of GPA, HPA and VPA between different sampling points' height are provided in Table 1.

From Figure 3, the best theoretical accuracy is obtained at the centre of beacons. It implies the target around the centre of the beacons can achieve more accurate positioning than those nearby the beacons. From Figure 4, it suggests that the better positioning region in three-dimensional scenarios is also like a square, similar to the beacon formation. Therefore, the beacons for acoustic positioning should be located beyond the interesting region. What's more, the better performance will be achieved when the distances between beacons are larger, only if the target could receive the signals from all beacons. Over the interesting region, GPA show ideal accuracies in some parts of the interesting region. This fact will be of great importance to determine the number of beacons needed or whether the positioning accuracy over a given area meets user needs.

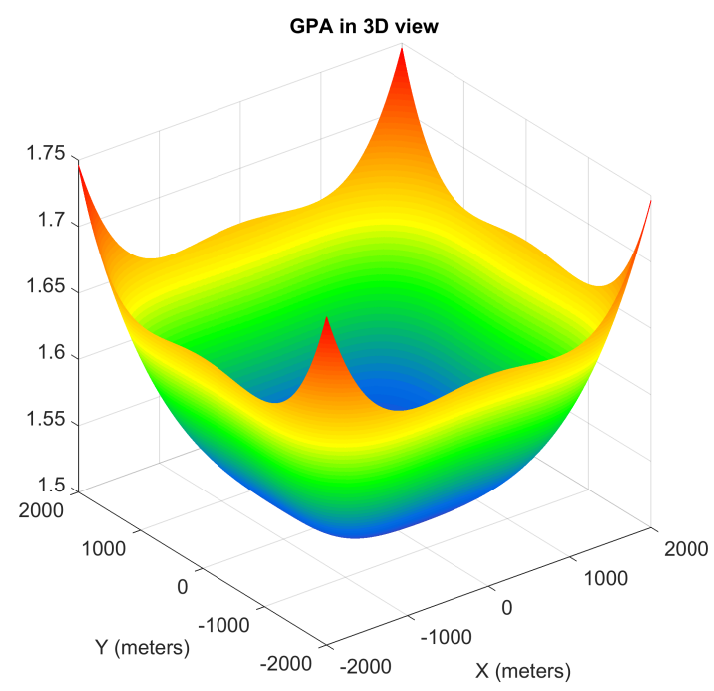

Figure 3. Level curves of HPA in three-dimensional scenarios. 


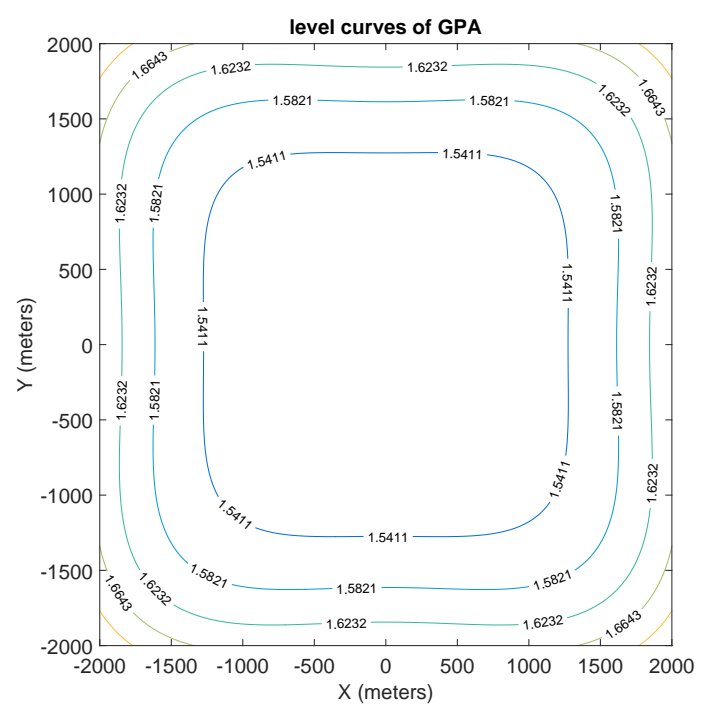

Figure 4. Level curves of HPA in three-dimensional scenarios.

For each case in three-dimensional scenarios, the minimum and maximum GPA, HPA as well as VPA are computed with the optimal beacon placement. The results are shown in Table 1.

Table 1. Results for three different sampling points' height.

\begin{tabular}{ccccccc}
\hline & GPAmin & GPAmax & HPAmin & HPAmax & VPAmin & VPAmax \\
\hline$-1000 \mathrm{~m}$ & 1.5651 & 1.9538 & 1.0607 & 1.2862 & 0.9682 & 1.5811 \\
$-2000 \mathrm{~m}$ & 1.5000 & 1.7464 & 1.2247 & 1.4491 & 0.8602 & 1.0000 \\
$-3000 \mathrm{~m}$ & 1.6116 & 1.9274 & 1.4577 & 1.6748 & 0.6872 & 0.9539 \\
\hline
\end{tabular}

It's obvious shown that minimum of GPA(GDOP) is obtained when the sampling points' height is $-2000 \mathrm{~m}$, and this value satisfied the formula $3 / \sqrt{n}=3 / \sqrt{4}=1.5$. What's more, the data in Table 1 imply that HPA(HDOP) grow proportional to the height of sampling points. However, VPA(VDOP) grow inversely proportional to the height of sampling points. This conclusion is consistent with the theory in section 3.2.

\section{Conclusions}

This paper offered a new characterization of the solutions to assess and optimize acoustic positioning system. By assuming that the range measurements between the sampling points and the acoustic beacons were corrupted by white Gaussian noise, the assessment parameter DOP related to the positioning accuracy were derived. Then the best positioning accuracy to be obtained was converted into that of minimizing the GDOP conveniently. Furthermore, unlike other work only use GDOP to optimize the formation and cannot assess the performance of any specified dimensions whether users satisfy, we use GPA, HPA and VPA to assess the optimal geometric beacon formation in each dimension for any point in three-dimensional space. This new assessment model can provide users with guidance advices to optimize performance of each specified dimension. Finally, numerical simulations support the view that the methodology proposed to estimate performance of acoustic system is feasible. Future work will aim at: 1) extending the methodology developed to deal with time bias errors; 2) studying the performance of the acoustic positioning system on condition that the beacons is in motion with ocean currents. 
Acknowledgments: This work is supported by National Natural Science Foundation of China (No. 61633008, No. 61374007, No. 61601262, No.61701487), Natural Science Foundation of Heilongjiang Province of China (No. F2017005) and Natural Science Foundation of Hainan Province of China (No. 117212, No.417211).

Author Contributions: X.C. wrote the paper; L.Z. and X.C. conceived and designed the experiments; Y.H. and C.L. performed the simulations; C.L. and L.Y. analyzed the data. All authors read and approved the final manuscripts. Conflicts of Interest: The authors declare no conflict of interest.

\section{References}

1. Xu, J. N.; He, H. Y.; Qin, F. J.; Chang, L. B. A novel autonomous initial alignment method for strapdown inertial navigation system. IEEE Transactions On Instrumentation And Measurement 2017, 66, 2274-2282, 10.1109/TIM.2017.2692311.

2. Chang, L. B.; Li, Y.; Xue, B. Y. Initial alignment for a doppler velocity log-aided strapdown inertial navigation system with limited information. IEEE-ASME Transactions On Mechatronics 2017, 22, 329-338, 10.1109/TMECH.2016.2616412.

3. Rice, H.; Kelmenson, S.; Mendelsohn, L. Geophysical Navigation Technologies And Applications. In Proceedings of the Position Location and Navigation Symposium, Monterey, CA, USA, April 2006; pp. 618-624.

4. Teixeira, F. C. Novel Approaches To Geophysical Navigation Of Autonomous Underwater Vehicles. In Proceedings of the International Conference on Computer Aided Systems Theory, Las Palmas de Gran Canaria, Spain, February 2013; pp. 349-356.

5. Bonin-Font, F.; Ortiz, A.; Oliver, G. Visual navigation for mobile robots: A survey. IEEE-ASME Transactions On Mechatronics 2008, 53, 263-296, 10.1007/s10846-008-9235-4.

6. Eustice, R.; Pizarro, O.; Singh, H. Visually augmented navigation for autonomous underwater vehicles. IEEE Journal of Oceanic Engineering 2008, 33, 103-122, 10.1109/JOE.2008.923547.

7. Cheng, X. Z.; Shu, H. N.; Liang, Q. L. Silent positioning in underwater acoustic sensor networks. IEEE Transactions On Vehicular Technology 2008, 57, 1756-1766, 10.1109/TVT.2007.912142.

8. Bayat, M.; Crasta, N.; Aguiar, A. P.; Pascoal, A. M. Range-Based Underwater Vehicle Localization in the Presence of Unknown Ocean Currents: Theory and Experiments. IEEE Transactions On Control Systems Technology 2016, 24, 122-139, 10.1109/TCST.2015.2420636.

9. Zhang, T.; Chen, L. P.; Li, Y. AUV Underwater Positioning Algorithm Based on Interactive Assistance of SINS and LBL. Sensors 2016, 16, 1-22, 10.3390/s16010042.

10. Shabani, M.; Gholami, A.; Davari, N. Asynchronous direct Kalman filtering approach for underwater integrated navigation system. Nonlinear Dynamics 2014, 80, 71-85, 10.1007/s11071-014-1852-9.

11. Hong, Z. Two-Dimensional Optimal Sensor Placement. IEEE Transactions on Systems, Man, and Cybernetics 1995, 25, 781-792, 10.1109/21.376491.

12. Levanon N. Lowest GDOP in 2-D scenarios. IEE Proceedings - Radar Sonar And Navigation 2000, 147, 149-155, 10.1049/ip-rsn:20000322.

13. Rob, C.; Ronald, C.; Christopher J. H.; etc. Performance of stand-alone GPS. In Understanding GPS - Principles and Applications, 2nd ed.; Elliott D. K., Christopher J. H., Artech House: London, UK, 2006; pp. 322-328, ISBN 1-58053-894-0.

14. Moreno-Salinas, D.; Pascoal, A. M.; Aranda, J. Optimal Sensor Placement for Multiple Target Positioning with Range-Only Measurements in Two-Dimensional Scenarios. Sensors 2013, 13, 10674-10710, $10.3390 /$ s130810674.

15. Sonia, M.; Francesco, B. Optimal sensor placement and motion coordination for target tracking, Automatica. Automatica 2006, 42, 661-668, 10.1016/j.automatica.2005.12.018.

16. Moreno, D.; Pascoal, A. M.; Alcocer, A.; Aranda, J. Optimal Sensor Placement for Underwater Target Positioning with Noisy Range Measurements. In Proceedings of the 8th IFAC Conference on Control Applications in Marine Systems, Rostock, Germany, September 2010; pp. 85-90.

17. Moreno-Salinas, D.; Pascoal, A. M.; Aranda, J. Optimal sensor placement for underwater positioning with uncertainty in the target location. In Proceedings of the IEEE International Conference on Robotics and Automation, Qingdao, China, September 2011; pp. 2308-2314. 
18. Moreno-Salinas, D.; Pascoal, A. M.; Aranda, J. Sensor Networks for Optimal Target Localization with Bearings-Only Measurements in Constrained Three-Dimensional Scenarios. Sensors 2013, 13, 10386-10417, $10.3390 / \mathrm{s} 130810386$.

19. Moreno-Salinas, D.; Pascoal, A. M.; Aranda, J. Optimal Sensor Trajectories for Mobile Underwater Target Positioning with Noisy Range Measurements. In Proceedings of The 19th World Congress of the International Federation of Automatic Control, Cape Town, South Africa, August 2014; pp. 5139-5144.

20. Moreno-Salinas, D.; Pascoal, A. M.; Aranda, J. Optimal Sensor Placement for Acoustic Underwater Target Positioning With Range-Only Measurements. IEEE Journal of Oceanic Engineering 2016, 41, 620-643, 10.1109/JOE.2015.2494918.

21. Zou, Y.; Wang, C.; Zhua, J.; Lia, Q. Optimal sensor configuration for positioning seafloor geodetic node. Ocean Engineering 2016, 142, 1-9, 10.1016/j.oceaneng.2017.06.033.

22. Wang, W.; Lv, C.C.; Li, X. Assessment Models and Rules of GNSS Interoperability. In International Conference on Mechatronics and Semiconductor Materials, Xian, China, September 2013; pp. 808-811.

23. Rajasekhar, C.; Dutt, V. Rao, G. Investigation of best satellite-receiver geometry to improve positioning accuracy using GPS and IRNSS combined constellation over Hyderabad region. Wireless Personal Communications 2016, 88, 385-393, 10.1007/s11277-015-3126-3.

24. Swaszek, P. F.; Hartnett, R. J.; Seals, K. C. Lower Bounds on DOP. Journal Of Navigation 2017, 70, 1041-1061, 10.1017/S0373463317000248. 